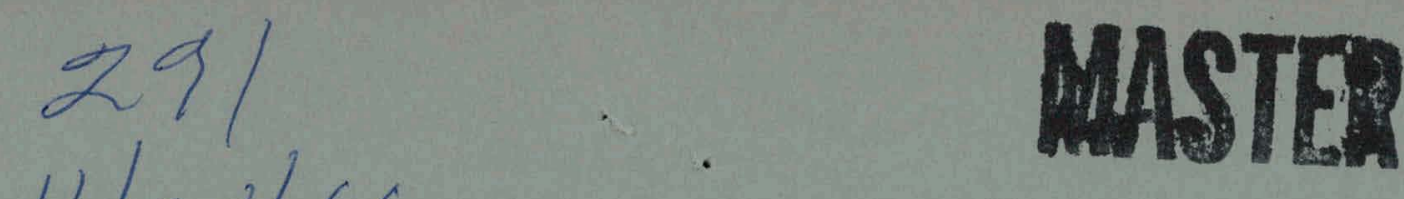

\title{
REPORT ON A SPARK CHAMBER READER SYSTEM
}

\section{SidNEy HeLler AND GeORGE SCHWENDER}

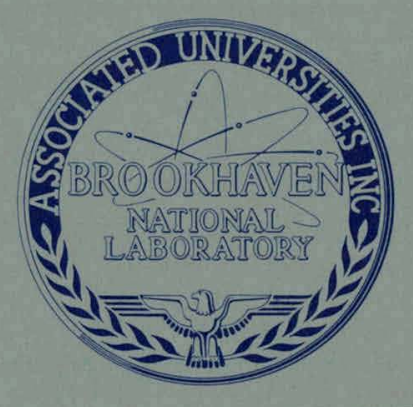

RELEASED FOR ANNOUNCEMENT

IN NUCLEAR SCIENCE ABSTRACTS

November 1965

BROOKHAVEN NATIONAL LABORATORY

ASSOCIATED UNIVERSITIES, INC.

under contract with the

UNITED STATES ATOMIC ENERGY COMMISSION 


\section{DISCLAIMER}

This report was prepared as an account of work sponsored by an agency of the United States Government. Neither the United States Government nor any agency Thereof, nor any of their employees, makes any warranty, express or implied, or assumes any legal liability or responsibility for the accuracy, completeness, or usefulness of any information, apparatus, product, or process disclosed, or represents that its use would not infringe privately owned rights. Reference herein to any specific commercial product, process, or service by trade name, trademark, manufacturer, or otherwise does not necessarily constitute or imply its endorsement, recommendation, or favoring by the United States Government or any agency thereof. The views and opinions of authors expressed herein do not necessarily state or reflect those of the United States Government or any agency thereof. 


\section{DISCLAIMER}

Portions of this document may be illegible in electronic image products. Images are produced from the best available original document. 
BNL 969 (T-407)

(Mathematics and Computers - TID-4500)

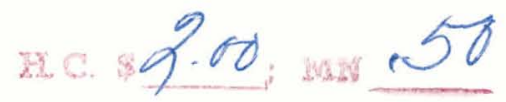

\section{REPORT ON A SPARK CHAMBER READER SYSTEM}

\section{Sidney Heller and George SChWEnder}

This report was prepared as an account of Government sponsored work. Neither the United States, nor the Commission, nor any person acting on behalf of the Commission:

A. Makes any warranty or representation, expressed or implied, with respect to the accuracy, completeness, or usefuiness of the information contained in this report, or that the use of any information, apparatus, method, or process disclosed in this report may not infringe privately owned rights; or

B. Assumes any liabilities with respect to the use of, or for damages resulting from the use of any information, apparatus, method, or process disclosed in this report.

As used in the above, "person acting on behalf of the Commission" includes any employee or contractor of the Commission, or employee of such contractor, to the extent that such employee or contractor of the Commission, or employee of such contractor prepares, dich eninates, or provides a with the Commission, or his employment with such contractor.

\section{BROOKHAVEN NATIONAL LABORATORY \\ UPTON, NEW YORK 11973}




\section{LEGAL NOT I C E}

This report was prepared as an account of Government sponsored work. Neither the United States, nor the Commission, nor any person acting on behalf of the Commission:

A. Makes any warranty or representation, expressed or implied, with respect to the accuracy, completeness, or usefulness of the information contained in this report, or that the use of any information, apparatus, method, or process disclosed in this report may not infringe privately owned rights; or

B. Assumes any liabilities with respect to the use of, or for damages resulting from the use of any information, apparatus, method, or process disclosed in this report.

As used in the above, "person acting on behalf of the Commission" includes any employee or contractor of the Commission, or employee of such contractor, to the extent that such employee or contractor of the Commission, or employee of such contractor prepares, disseminates, or provides access to, any information pursuant to his employment or contract with the Commission, or his employment with such contractor.

\section{PRINTED IN USA}

PRICE $\$ 2.00$

Available from the

Clearinghouse for Federal Scientific and Technical Information

National Bureau of Standards

U.S. Department of Commerce Springfield, Virginia 


\begin{abstract}
This report provides a description of a spark chamber reader system available at Brookhaven National Laboratory. A general description of the scanner, giving its salient features and numerical values, is followed by sections detailing the available programs for the system, the user requirements, and the system limitations. The appendix contains a description of a calibration procedure for the digitizer.
\end{abstract}




\section{REPORT ON A SPARK CHAMBER READER SYSTEM}

\section{INTRODUCTION}

The Spark Chamber Reader is an automated system for optically scanning $35-\mathrm{mm}$ film. ${ }^{1}$ Two scanning positions are incorporated - one produces a normal scan and the other an orthogonal scan. The scans are accomplished with the aid of 16 lenses mounted in a rotating wheel. As each lens passes across a cone of light produced by a pinhole and a mercury arc lamp, a light spot is generated that sweeps across the film. The light passes through the film and is then monitored by a photomultiplier tube at each window, for the determination of the data points. A data level control is provided to compensate for different grades of film.

Each frame of film is metered precisely to one window, digitized, and then metered again for the digitization in the second window. The machine is flexible and can produce either a single or double scan, as required.

After metering to a window, the stage carrying the windows is moved at a uniform rate to produce an axis of motion. The movement of the light beam produces the second axis of motion.

When a data point is recognized, the $x$ address is placed in the core memory, and after completion of the scan the $y$ address is also recorded. These data are then transferred in groups of 128 words to magnetic tape for processing by the IBM 7094 computer. The computer programs are designed to analyze the data and recognize linear tracks.

The system has facilities for incorporating indicative data and can handle machine malfunctions or overflows on its data handling capabilities. In addition, it has a preselection mode of operation. ${ }^{2}$

\section{Preselector}

This unit of equipment consists of a film transport, a viewing screen, a paper punch, and frame registration and frame and reel number sensing circuits.

The operator, when viewing the frame, makes the decision as to its usefulness. If the information is useful, a readout of its frame and reel number is recorded on punched paper tape. Upon completion of this operation, the film and punch paper can be mounted on the digitizer. The digitizer then searches for the addresses as called for and digitizes only those frames. This entire preselection mode of operation may be omitted; film can be placed directly on the digitizer and each frame digitized.

\section{GENERAL DESCRIPTION}

\section{Optical System}

The layout of the optical components is shown isometrically in Figure 1. A fixed, illuminated pinhole is demagnified by a factor of 10 onto the film; motion of the lenscs produces the scan line. Sixteen microscope objectives with large numerical apertures are placed around the circumference of a rotating disk with their optical axes parallel to the axis of the disk. As long as an objective is passing through the cone of light emanating from the pinhole, it focuses a spot of light on the film.

There are three independent optical channels; two provide for the normal and the orthogonal scans of the film. The third channel focuses on a transmission grating which provides the $x$ coordinate of the scan by digitizing the position of the disk. A high-pressure mercury lamp is the source of light for all channels. In each, the light is collected and made parallel by a $210-\mathrm{mm} f / 3.5$ lens. This is followed by a $150-\mathrm{mm} f / 2.3$ lens which converges the light on the pinholes and demagnifies the $300-\mu^{2}$ arc to about $200 \mu^{2}$. 


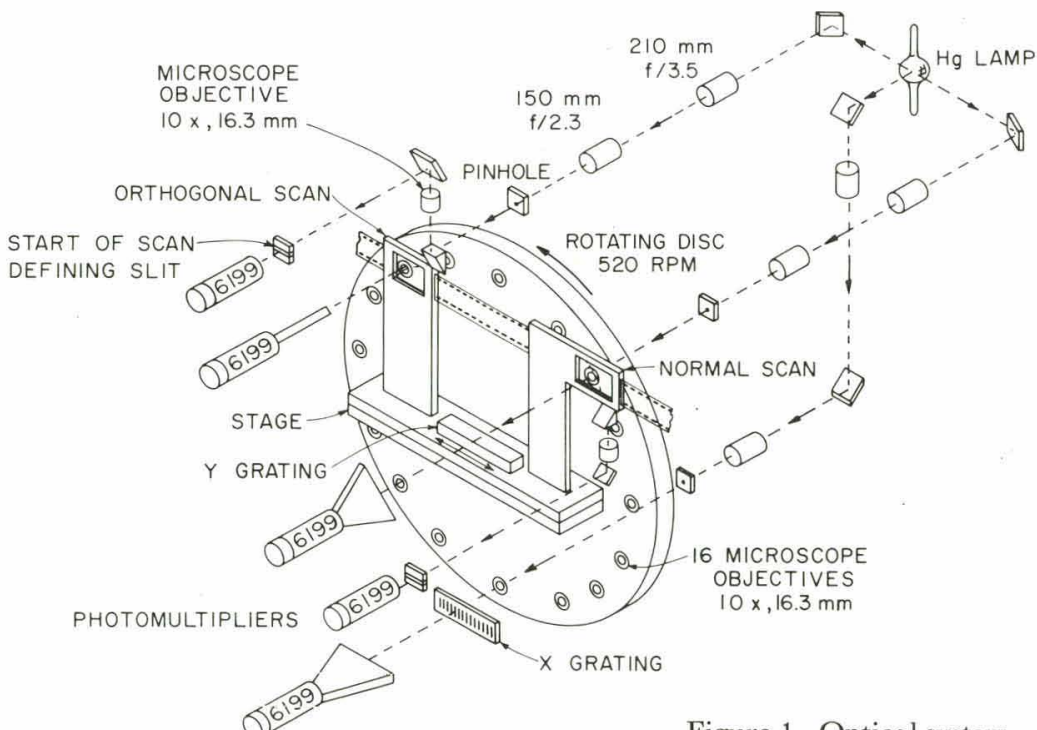

Figure 1. Optical system.

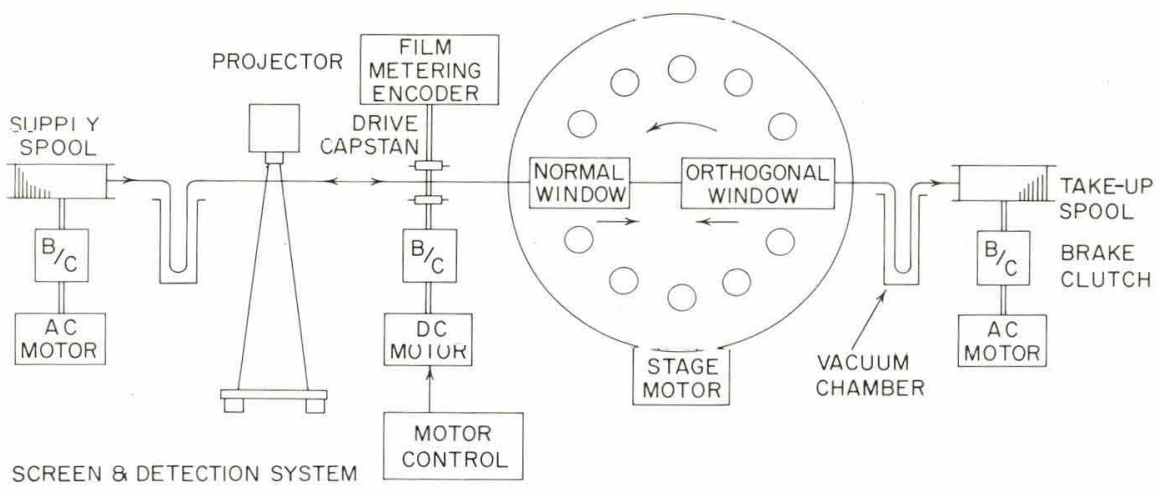

FOR FRAME REGISTRATION

\&. REEL \& FRAME NUMBER

Figure 2. Film transport system.

The two scanning positions are located so that the microscope objective rotates $45^{\circ}$ to the edge of the film; the normal and orthogonal scans are perpendicular to each other.

The $y$ motion is obtained from a precision stage to which the film is clamped by a vacuum platen. It is digitized by a Ferranti Moiré Fringe Grating System. The combination of the $x$ and $y$ motions produces a cycloidal projectory of the spot on the film.

The start of digitization for a particular scan is a signal produced by the spot making the scan. Just before the spot starts onto the film it is interrupted by a deflection $45^{\circ}$ silvered prism, and the spot is remagnified to the original pinhole size. The spot then passes over a slit and generates a startof-scan pulse. Light intensity is sensed in all three channels by photomultiplier tubes.

The disk is driven by a belt powered by a synchronous motor at $695 \mathrm{rpm}$. One scan line is generated every $5.4 \mathrm{msec}$, with a spot actually scanning for a period of $4 \mathrm{msec}$.

\section{Film Transport System}

The film transport system, shown schematically in Figure 2, handles 35-mm perforated film. The supply and take-up spools are buffered from the rest of the system by vacuum columns. They are driven by continuously running motors which are clutched in and out on command from pressure switches on the columns. Between the columns the film is driven by a sprocket drive capstan which, in turn, is driven by a reversible motor through an electrical brake-clutch assembly. 


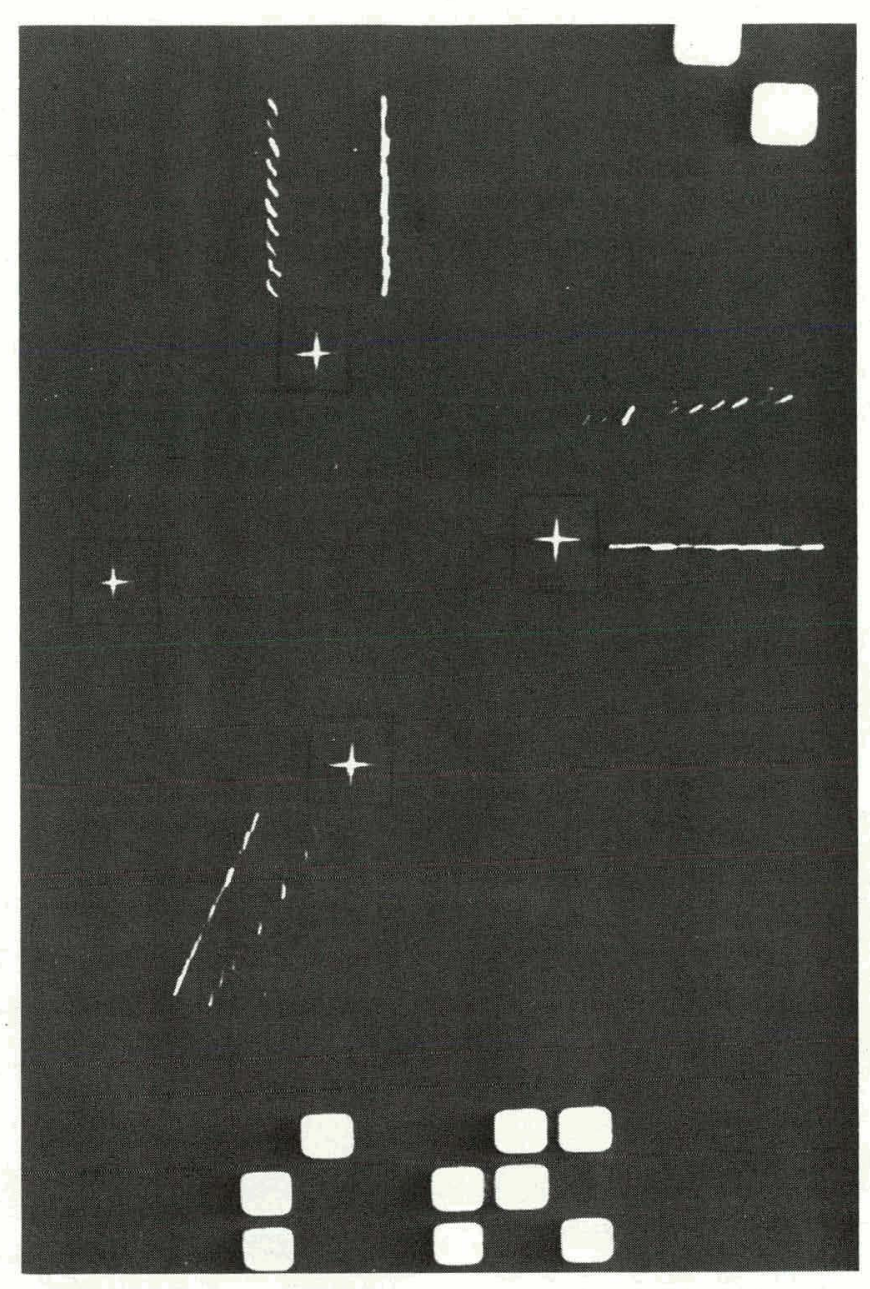

Figure 3. Photograph of spark chamber, showing registration boxes and frame and reel number matrix.

The film motion sequence starts when a frame passes through the projector. Frame registration marks are sensed by a matrix of photodiodes on the screen. At this time metering of the film is commenced through the rotary encoder mounted on the drive capstan shaft. The film is metered to a precise distance (the normal window), where it is clamped to the stage, and the normal scan of the frame is begun. When this is completed the film is released and advanced to the orthogonal window, where it is clamped once again, and the stage motor starts the driving of the stage in the reverse direction. Upon completion of the orthogonal digitization, the film is unclamped and the capstan motor returns the film to a position a half frame width ahead of the projector. The film is advanced again until the next frame is registered and the process is repeated.

The film travels at two variable, independent speeds. In the advanced mode its speed is $\approx 20$ $\mathrm{cm} / \mathrm{sec}$, and in the reverse mode, $\approx 43 \mathrm{~cm} / \mathrm{sec}$.

\section{Spark Chamber Format}

A typical format for a spark chamber photograph is shown in Figure 3. The two squares in the upper right-hand corner are the frame registration marks. The coding at the bottom gives the frame and reel number in a binary coded decimal system. These are read by the photodiodes on the screen at the instant that frame registration marks are sensed, and are placed in the core memory. The relative positions of the photodiodes can be adjusted. 


\section{Photomultiplier Output}

The signals directly at the output of the various photomultipliers are shown by the oscilloscope traces in Figure 4. The parameters for these traces were as follows: disk speed, $520 \mathrm{rpm}$; all pinholes, $55 \mu$ (this yielded a spot size of $7.5 \mu$ and a stage speed of $60 \mu /$ scan). The $x$ grating used had a $17-\mu$ cycle with the clear and opaque portions approximately equal at $8.5 \mu(3000 / \mathrm{in}$.).

Since these traces were recorded, both the wheel speed and stage speeds have been changed, as noted later, but the wave forms are typical.

The upper left-hand oscilloscope pattern is the $x$ grating signal at two different sweep speeds. The upper trace shows the entire passage of one spot across the grating. The individual crossings of the grating lines are not visible, but this envelope shows a light modulation of $\approx 75 \%$. The shrinkage
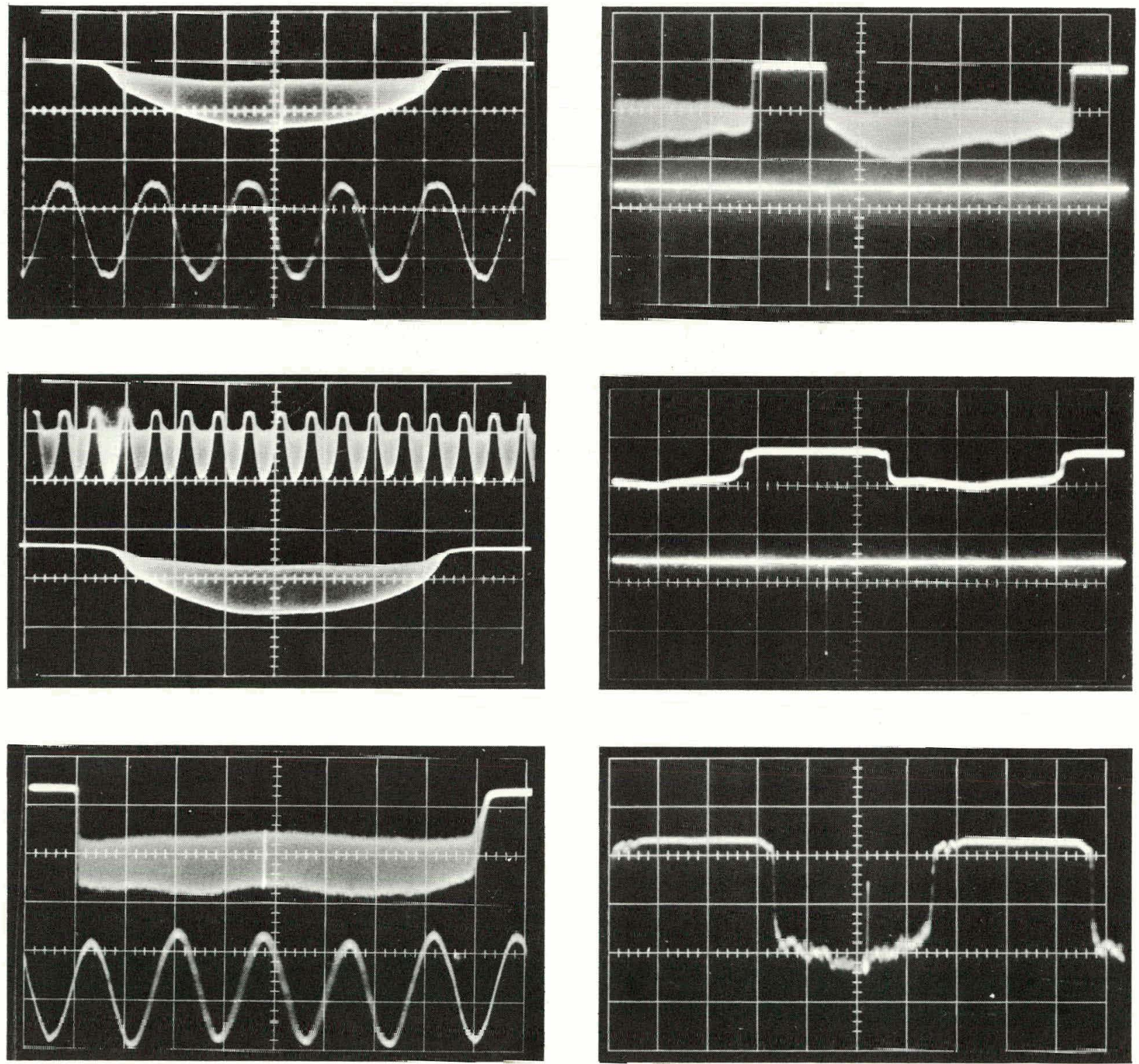

Figure 4. Oscilloscope traces of photomultiplier signals. 
of light intensity to either side of the center is caused by the vignetting of the light by the microscope lenses and some defocusing of the light spot. The lower trace is a portion of the upper one with a sweep speed sufficiently fast to show the individual crossings The mid-left photograph is again the $x$ grating, but with the scans produced by all 16 objective lenses. These first two patterns were taken with $85 \%$ of the available light removed by a uniform neutral density filter placed over the photomultiplier.

To remove the effect of vignetting, the uniform filter was replaced by a nonuniform one, and the $x$ grating signal shown at the lower left in Figure 4 was obtained. In the upper right trace the startof-scan is shown just below the $x$ grating pulse. The next pattern again shows the start-of-scan pulse, and above it the output of the photomultiplier behind the film platen when no film is in place. The last pattern was taken with film in place and one data point on the scan.

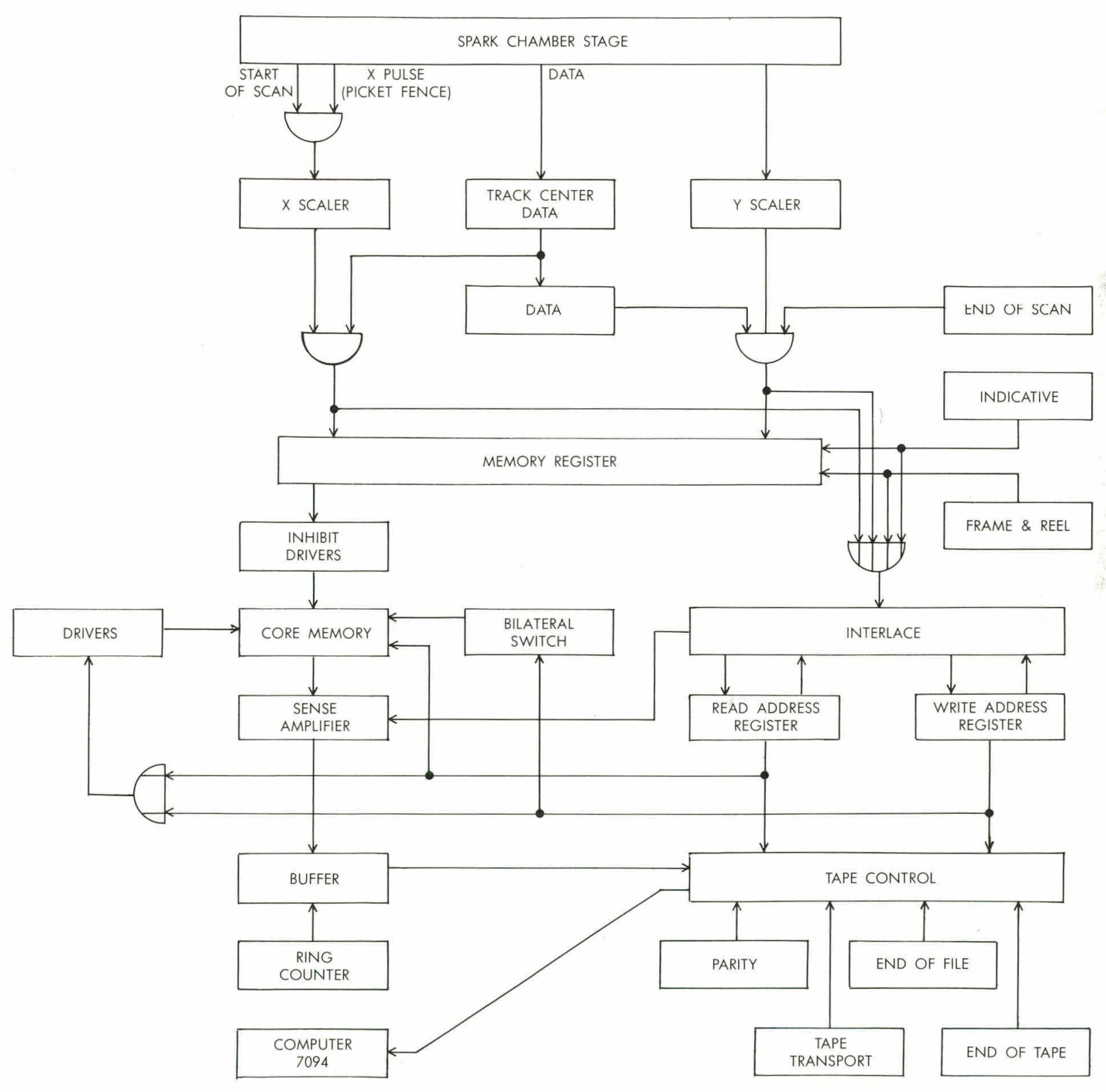

Figure 5. Diagram of the logic for the data processing unit. 


\section{Clock and Automatic Data Level Circuits}

The address of data is dependent upon the passage of the spot across the grating. It has been found that dirt, dust, etc., will cause the loss of counts. To eliminate this condition a clock circuit has been added that has a free running period greater than the grating frequency. The clock is synchronized to the grating. A single, double, or triple loss at the grating source is automatically corrected by the clock. Notification of this action is given to the machine operator.

To compensate for the differences in film background and developing rates an automatic bias circuit has been incorporated. This maintains the ratio fixed by the operator between data vs light (no data on the sensing circuits).

\section{Data Processing Unit}

One group of functions of the data processing unit is to register the start-of-scan and the $x, y$, and data pulses from the photomultiplier tubes, and to store and then write these coordinates on magnetic tape. Second, the unit commands and supervises all stage control functions, such as the movement and clamping of film and the starting and stopping of the capstan and stage motors, and monitors all safeguard checks of the machine. Third, it provides for the insertion of indicative information, gives the command for the search mode, and provides for preselection of frame and reel numbers that are to be digitized.

Figure 5 is an abbreviated block diagram of the logic for the data processing unit. Starting at the upper left-hand side of the block diagram the $x$ pulses, derived via the picket fence, are gated into the $x$ scaler after the start-of-scan has occurred. If a data point is detected during the scan it initiates the track centering circuit. This then gates the contents of the $x$ scaler into the memory register and sets the data flip-flop. At the completion of a scan the end-of-scan pulse takes the contents of the $y$ scaler and deposits this information in the memory register.

\section{Memory Register}

The memory register can receive four types of information: the coordinate of the scan ( $x$ scaler), the stage motion ( $y$ scaler), indicative information, and the frame and reel number. Indicative information may be placed on the tape by a series of switches at the console and will be at the head of all data particular to a file; it may have any format. The fourth input, the frame and reel number, is a bookkeeping function. Upon receipt of any one of the four inputs to the memory register, the interlace is notified of a write command. The interlace controls the function of reading or writing on the core, which is a 256-word, 18-bit unit.

\section{Write Cycle}

On a write command the interlace circuit receives the initial request. The write-address register is stepped by one unit. Then the decoding of the bilateral switches and the activation of the write driver and bilateral switch take place. Depending upon the state of the memory register, the inhibit winding is activated and zero is deposited in the core, or it is not activated and one is deposited in the core.

\section{Read Cycle}

After writing 128 words, the write-address register makes a request via the interlace for the initiation of the read-out. It also sends a command to the tape control to start the magnetic tape system. The interlace steps the read-address register, which is decoded for setting up the bilateral switches and the read drivers. The interlace also produces a strobe for sensing the information in the core and depositing it, via the sense amplifier, on a magnetic core buffer. At this point the core buffer places this information, via the tape control circuitry, on the magnetic tape. This loop continues until 128 words have been read out of the core. The read-address register then sends a stop command to the tape control unit and informs the interlace circuit. Provision is made within the tape control for 

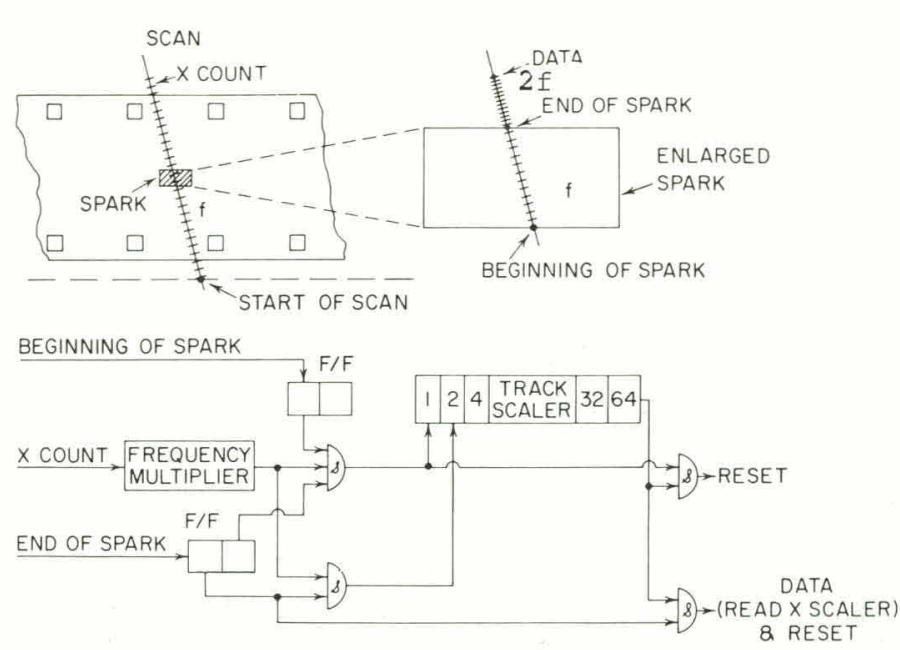

Figure 6. Track centering logic.

the production of a parity character, a longitudinal redundancy check character, an end-of-file character, and an end-of-tape character. This tape then has, for a particular frame digitization, many records of 128 words. The file itself has, within the first record, indicative information, if required, followed by frame and reel number, then the digitization of the normal window immediately followed by the digitization of the orthogonal window, the end-of-file, and, when required, the end-oftape indication.

\section{Track Centering}

The data pulse does not appear at the moment the spark is crossed, but at some constant distance beyond its center. The method by which the center of the spark is located is shown in Figure 6 . An independent track-centering scaler is used; when it is full, the overflow pulse causes the data point to be read or deleted, depending on the spark's width. The scaler is fed frequency $f$ while the spot is traversing the spark, and at twice the normal frequency thereafter; thus the distance from track center to data point is independent of spark width. The capacity of the track-centering scaler can be adjusted to match the maximum spark width expected. Should the spark width exceed the capacity of the scaler, no data are read.

\section{Machine Structure}

Figures 7 and 8 are two views of the machine.

\section{COMPUTER PROGRAMS}

A set of routines that can analyze the magnetic tape input data produced by the digitizer and can recognize linear tracks has been written, tested, and debugged. The frame of film may have any number of arbitrarily positioned spark chambers, but the IBM 7094 programs restrict the analysis to rectangular parallel plate chambers (the gaps need not be equidistant). A complete description of the programs is given in ref. 3.

Figure 9 is a general flow chart of the program, with details omitted. The unpacking of data, transformations to rectangular coordinates, and fiducial findings are dependent on the digitizer characteristics and formats. The first word of a file following the indicative information is decoded to provide an 8-digit frame number identification, the possible indicative words and error signals are processed, and the data are unpacked and transformed in the main program. The main program also carries the monitor control or calls of other programs. Options exist to obtain a CRT playback of 


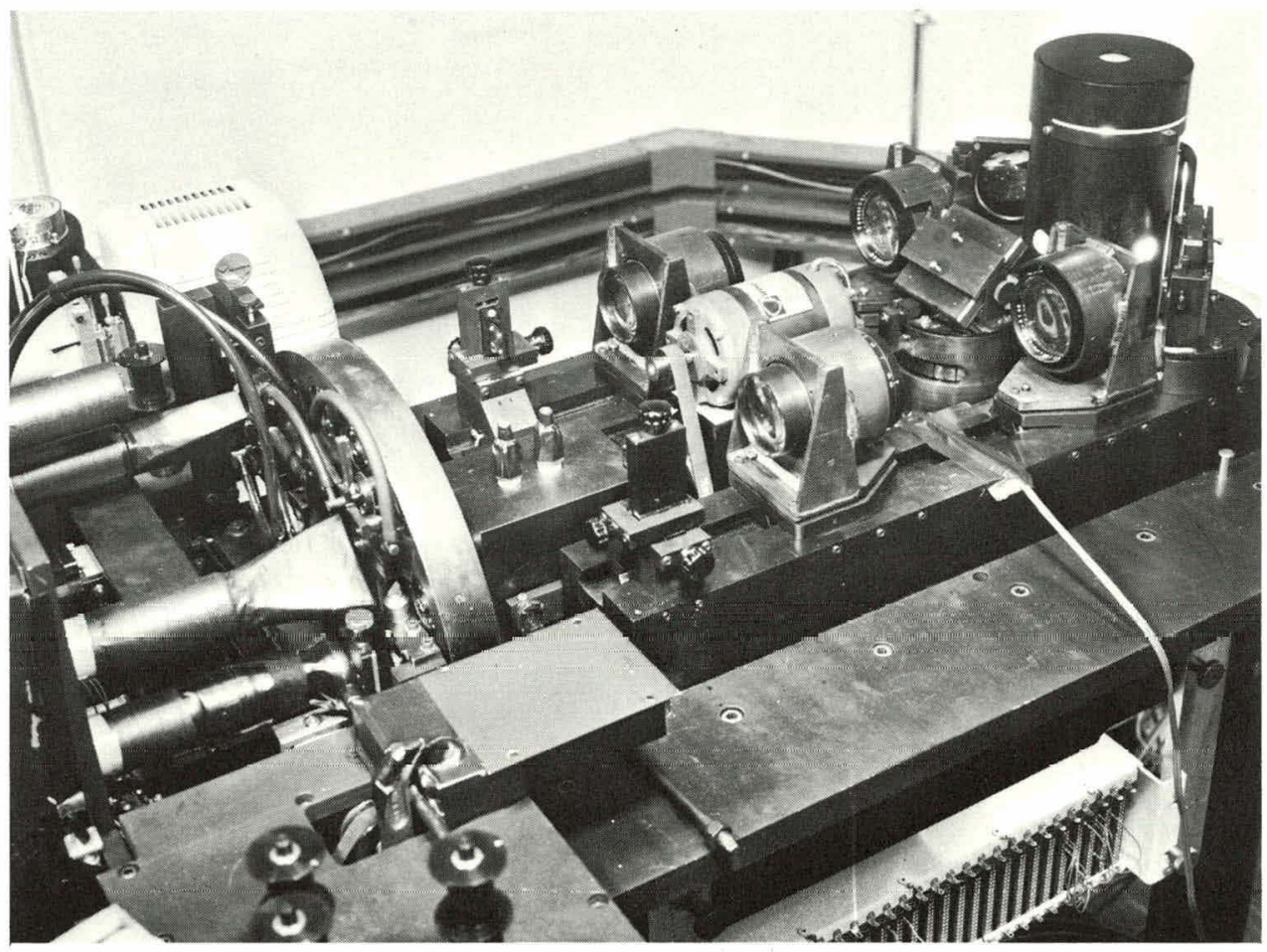

Figure 7. View of the digitizer, showing the optical apparatus.

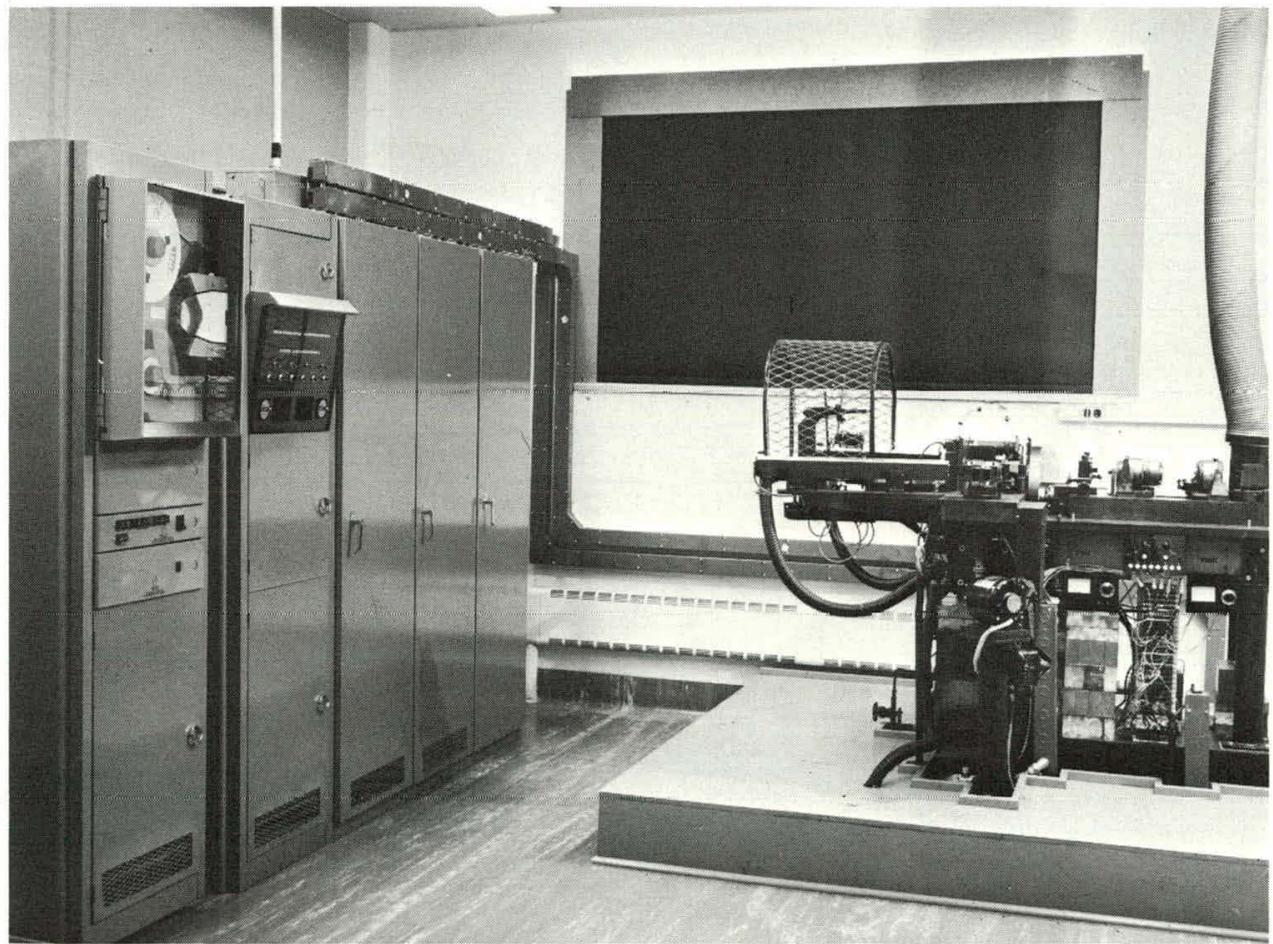

Figure 8. View of the spark chamber reader. 
digitized points. Any number of tapes may be processed. Each frame, with either one or two scans, represents a file on tape, and a double end of file indicates end of tape.

The fiducial finding routine FIDUC is among the first to be called. Any number of fiducials may be found with an equal number of calls of FIDUc. The transformation of three noncollinear fiducials into a corresponding given set of fiducials determines a rigid (or nearly rigid) motion by which all points are mapped into a fixed coordinate system. Hence all pictures for the same experiment have the same frame of reference independent of the skew or stop error introduced by film movement in the digitizer.
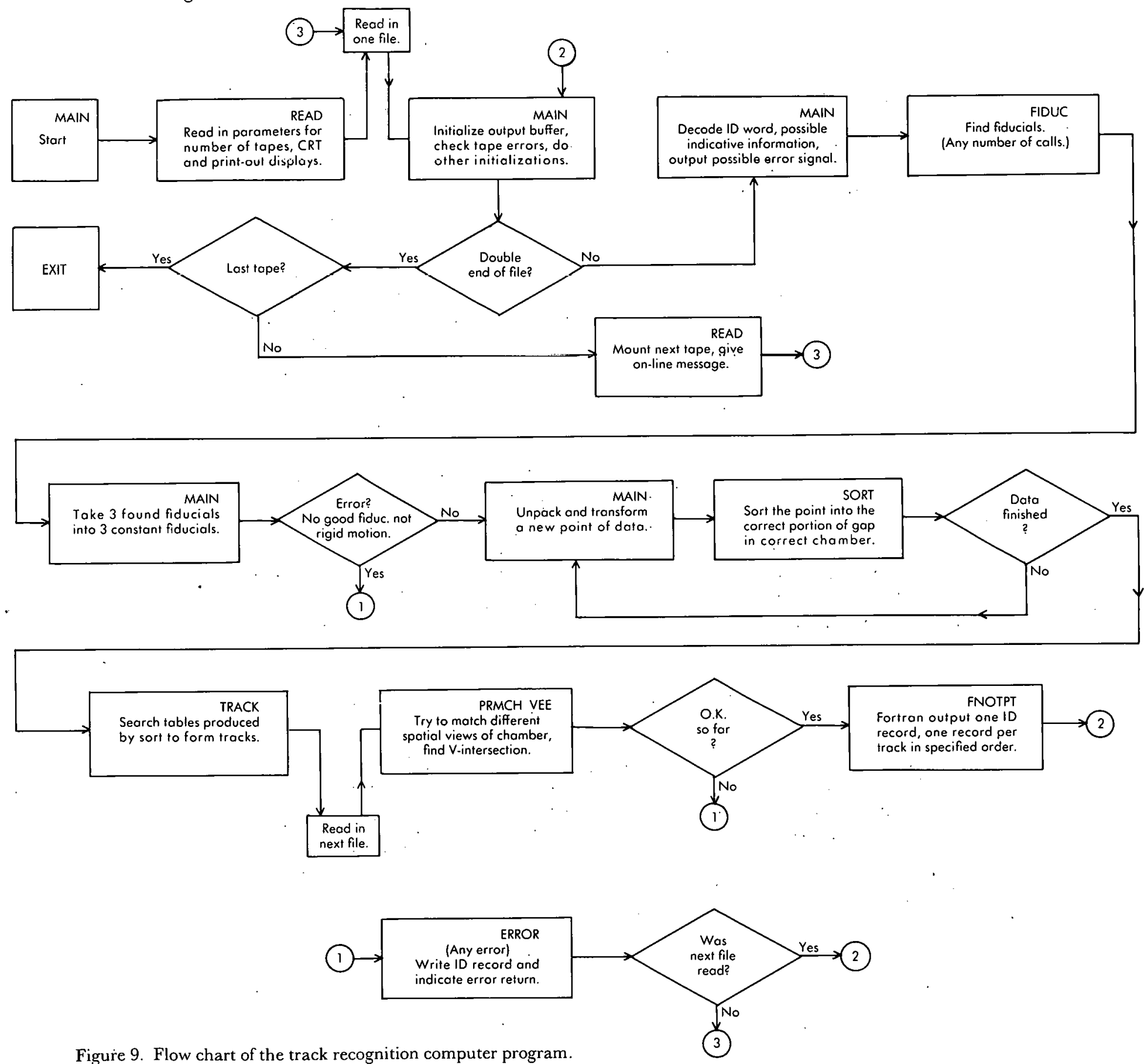

Figure 9. Flow chart of the track recognition computer program. 
Two scans of a picture are necessary if sparks occur in directions nearly parallel to both flyingspot arcs (i.e., at $45^{\circ}$ and $135^{\circ}$ to the horizontal). In this case each chamber is programmatically tagged for the proper scan, and the analysis is automatically handled by the software. One chamber should require only one scan, since all sparks occur in a direction approximately normal to the plates - even though the track direction may not be perpendicular to the gaps. Since either scan is tranisformed into the same coordinate system, the two scans are automatically merged when two scans are called for.

The subroutines are in closed form, which allows easy separation from the entire software package. As an example, a user may not be interested in the pattern recognition aspect of the programs. He can lift the main program, make trivial changes, and use it together with FIDUC as an independent package to produce rectangular coordinates of all digitized points.

The sORT and TRACK programs are logically independent of the flying-spot digitizer, although certain parameters are chosen with the criteria of the scanner in mind. For example, the scan-line pitch governs the average number of points digitized per spark, which in turn affects memory allocation. Certain spark-searching parameters cannot be set smaller than this pitch plus the minimum distance for distinct digitizations on a scan line. Accuracy and tolerance of other parameters is in part influenced by the initial accuracy of the digitization.

The rRMcrr routine is intended to match tracks in two spatial views of the same chamber (pictured as two chambers on film), while VEE looks for V-intersections of tracks within a chamber.

The usual output of the spark chamber programs is a list of tracks and their sparks for each chamber. An image of this is written on magnetic tape in FORTRAN compatible format, and an optional corresponding print-out is available. Any change in the type of output, e.g., from the tape to punch cards, or alteration of the logical flow of the program is the user's responsibility. Kinematical analysis and spatial reconstruction are also left to the user.

Among the utility routines of the system is a calibration routine VARY, which is concerned with the digitizer itself. This calibration has already been performed. It is necessary only to verify periodically that a standard grid film can be measured with sufficient accuracy using the parameters $R, L$, and $\alpha$ (see appendix) in the transformations to rectangular coordinates. First, the approximate fiducial areas of the cross marks must be input to the FIDUC program that is called by VARY; then, with the ranges and increments of $R, L$, and $\alpha$ narrowly set, VARY prints out the values of $R, L$, and $\alpha$ that allow the fiducial found to conform to the standard. These values should be invariant - if they have changed, an entire new calibration procedure must be performed. Such a procedure is fully described in the appendix.

\section{USER REQUIREMENTS}

\section{Fiducials}

Fiducial marks can be as many as needed for the experiment. Three of them should be noncollinear to form a triangle at least $20 \mathrm{~mm}^{2}$ in area. These three would be used as the constant fiducials to fix the rectangular coordinate system on film. The legs of the fiducials must be horizontal and vertical, with each leg at least $1 \mathrm{~mm}$ in total length. The fiducials should be clear of sparks or other data by at least $0.5 \mathrm{~mm}$, especially in the horizontal direction (direction in which the film moves in the scanner).

\section{Registration Boxes}

The logical buildup of this unit uses 5 photodiodes each spaced on $1 / 10$-in. centers. This requires that the individual box on the film be $1 \times 1 \mathrm{~mm}$. This also applies to the frame and reel number indicators. (See Figures 10 and 11.) 


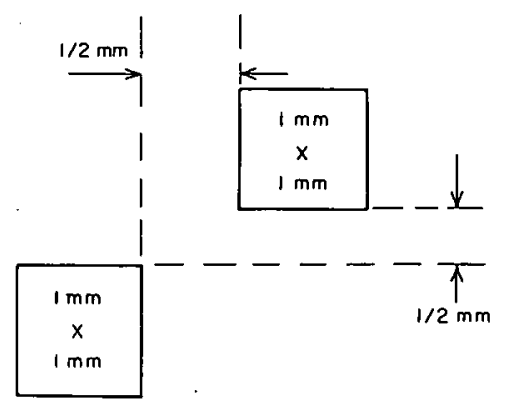

Figure 10. Registration box layout.

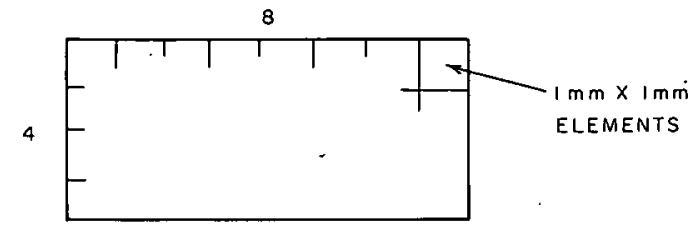

Figure 11. Frame and reel number layout.

\section{Photography}

It cannot be stressed enough that the quality of the negative is of prime importance. Scratches, dust, dirt, focus, and background all affect the operation and quality of the digital information produced by the machine. The useful area of the frame is $24 \times 34 \mathrm{~mm}$.

\section{Computer Program Inputs}

A detailed list of input parameters and input data is given in ref. 3. Briefly, these entail the following.

1. Fiducials: scanner coordinates of fiducials as they appear on film are input to the MAIN program call of the FIDUc routine. Rectangular coordinates of three of these fiducials to serve as reference fiducials are also supplied.

2. Survey of film event: the sorr routine requires the four corners of each chamber (with respect to an origin determined by the fixed fiducials), the position and direction of the gaps in:each chamber, and a decision as to which scan shall apply for a given chamber. An entire survey of all significant points on an actual frame of film by microscope or hand digitizer is very useful and convenient.

3. Parameters for the TRACK routine often depend on the experiment in question. Among them are the number of chambers, minimum and maximum number of points per spark, maximum number of gaps per chamber, number of sparks that can be skipped in a continuous track, minimum and maximum number of tracks in each chamber, track searching parameters such as road width from gap to gap, and various memory allocating parameters. (See ref. 3.)

4. Execution terminating conditions may be inserted by the user by altering the logical flow when certain conditions are not met. This usually requires more than parameter changes; any program patches added are the user's responsibility.

5. The exact number of items and arrangement of each for output on tape, as well as specifications for labeling of tracks for the use of the PRMCH routine, usually require a few days of coding.

6. Kinematical analysis and spatial reconstruction of events are not included in the system. 


\section{LIMITATIONS AND CRITERIA OF THE SYSTEM}

\section{Tape Transport:}

Write mode $=$ a non-return-to-zero system.

Density $=200$ bits $/$ in.

Speed $=60 \mathrm{in} . / \mathrm{sec}$.

Size of tape $=1 / 2$-in. IBM compatible format.

Channels $=6$ channels + parity.

\section{Scalers:}

$$
\begin{aligned}
& x \text { count }=4.233 \mu \text { (least count }), \text { or } 6000 / \text { in. } \\
& y \text { count }=10 \mu \text { (least count })
\end{aligned}
$$

\section{Optical System:}

Spot size $=7.5 \mu$ (this spot size can be readily increased or decreased by changing the pinhole size).

Word Format: See Figure 12.

Track Centering and Memory Cycle:

Write time $=7 \mu$ scc.

Track centering scaler $=7$ bits.

Lcast count of track centering scaler $=4.2 \mu$.

Maximum spark width $=4.2 \times 128(7$ bits $)=532 \mu$.

Maximum delay between data points $=2.21 \times 128=271 \mu$.

\section{Critical Dimensions:}

In the $-(2,0)$ scan,

$$
\begin{aligned}
& R=36,900 \times \text { units; } \\
& L=3470 x \text { units, } \\
& \alpha=45.0573^{\circ} ;
\end{aligned}
$$

In the $(6,4)$ scan,

$$
\begin{aligned}
& R=37,500 x \text { units, } \\
& L=4000 x \text { units, } \\
& \alpha=44.9427^{\circ},
\end{aligned}
$$

where $R$ is the radius of the flying-spot arc, $\alpha$ is the inclination of the scanner $x$-axis, $L$ is the distance on the scanner $x$ axis from the start of scan to the intersection of a.perpendicular. radius and the axis. (see Figure 13):

\section{Film System:}

Film speed forward $=20 \mathrm{~cm} / \mathrm{sec}$.

Film. speed reverse $=43 \mathrm{~cm} / \mathrm{sec}$.

Film reel can mount ân 800-ft spoul ư film.

Accuracy of stopping at a window position $= \pm 1 / 2 \mathrm{~mm}$.

Stage Speeds: The machine is at present set up for a stage speed of $30 \mu$ per scan line. Three other speeds, with their assorted pulleys, are available.

Time of Digitization: For a digitization requiring both scans with a stage speed of $30 \mu / \mathrm{scan}$, the total cycle time for the entire digitization and film metering is $29 \mathrm{sec} / \mathrm{frame}$.

Compufer Time: One file representing one frame with about 3000 data points takes $1 \mathrm{sec}$ for processing on the IBM 7094 computer.

Accuracy and Typical Results: For a digitization of a straight line and making a least-squares fit, the root mean squared deviation varies from 7 to $10 \mu$, depending on the position of the line; the average deviation is between 6 and $8 \mu$. A circle fit gives similar results with an rms of $8.5 \mu$ and an average deviation of $7 \mu$. Digitization of a single point with the stage still gives a maximum spread of $50 \mu$ between the 16 lenses, with a standard deviation of $8 \mu$. A standard grid, which has been used to calibrate the digitizer, shows relative errors of $<1$ in 1700 over distances of $15 \mathrm{~mm}$ or greater. 


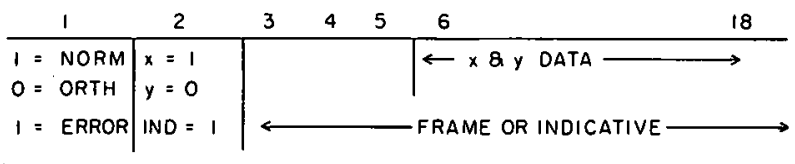

Figure 12. 18-Bit word.

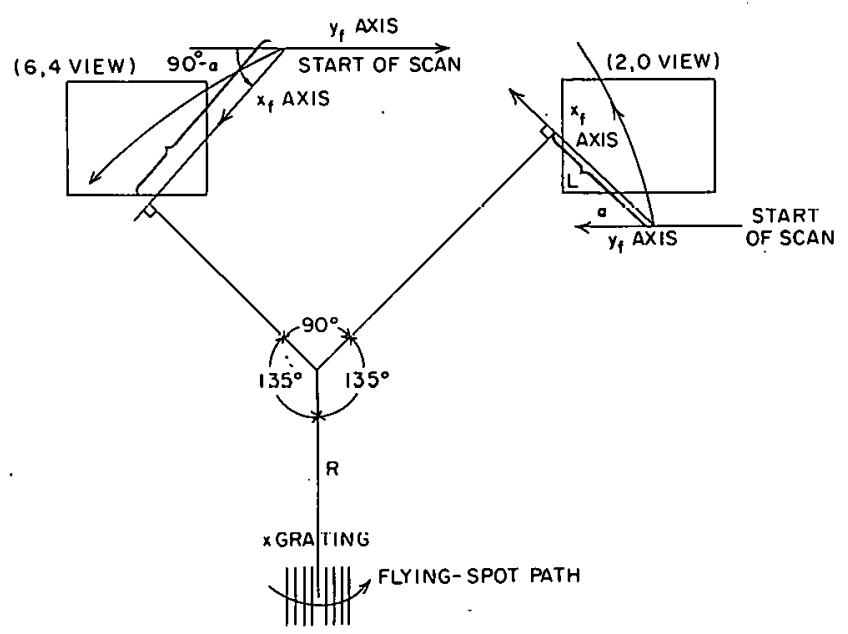

Figure 13.

\section{APPENDIX}

\section{Calibration Procedure}

Several measurements associated with the digitizer are not known, prior to calibration, with the precision needed for transformation to rectangular coordinates. These are

$R=$ radius described by the flying-spot arc. $R$ can be different in either window or on the $x$ grating.

$L=$ distance along a line (the $x_{f}$-axis) about $45^{\circ}$ to the horizontal (the $y_{f}$-axis) to the intersection with a perpendicular radius.

$\alpha=$ acute angle made by the $x_{1}$-axis with the $y_{r}$-axis.

An $x$ unit as measured along a $45^{\circ}$ line to the horizontal is affected by $R, L$, and $\alpha$ in a nonlinear fashion. $R$ is not unique if the focal planes of both viewing windows and the $x$ glass grating are not the same. If the lenses pass through a different part of the light cones in these three areas, $R$ will be different. $L$ is dependent on the positions of the start-of-scan slits in each of the viewing windows. The angle $\alpha$ is determined by the tilt of the $x$ glass grating to the horizontal ( $y_{1}$-axis). The corresponding angle in the other window is therefore $90^{\circ}-\alpha$. When the grating lines are perfectly vertical and $R$ is unique, an $x$ unit $=4.233 \mu(6000 x=1 \mathrm{in}$.). $R, L$, and $\alpha$ may be determined by the following steps:

1. Digitize a standard grid to produce both scans on a single file of magnetic tape.

2. From a binary dump of the tape, for each scan accurately determine 3 cross-mark fiducials whose centers form the vertices of a suitably large isosceles right triange (say, 15-mm legs). The method of finding the intersection is given below.

3. Determine the effective magnification factors $F_{1}, F_{2}$ for each scan.

4. With the knowledge of the magnification factors, make an initial guess for $R, L$, and $\alpha$. Run tape through a program called VARY (see ref. 1) which varies $R, L$, and $\alpha$ over a desired range with an 
arbitrary number of intervals and prints out only those triangles congruent to the standard to within a prescribed error. The $R, L$, and $\alpha$ of those in the output are the ones to use. If there are several sets to choose from, $\alpha$ in one window should be the complement of $\alpha$ in the other. It is also known, or can be determined by hardware tests, that $L$ in one window is a certain fixed distance larger than $L$ in the other scan.

5. If ambiguity still remains, other sets of three fiducials must be found and steps 2 to 4 repeated until the common set of values for $R, L$, and $\alpha$ is unique or localized within tolerable limits. Where such a repetition gives no common values of $R, L$, and $\alpha$, hardware trouble is to be suspected.

A separate check on local errors can be made by digitizing a straight line and computing the standard deviation (this can be done by hand directly from a binary dump) or by digitizing a circle and using the program CRCFT (see ref. 3 ) which finds deviations of points from a best-fit circle. The standard deviation should be $<10 \mu$.

\section{Determination of Grid Fiducials From a Binary Dump}

To find a fiducial cross mark on grid film the following procedure can be employed. Several $x$ levels can be detcrmincd by inspecting a binary dump; they represent horizontal grid lines. The vertical grid lines appear within a scan line as monotonic functions of a change in $x$, i.e., $\Delta x=$ $x_{\text {constant }}=x_{\text {vertical }}$ decreases monotonically as the scinl lines approach the cross-mark intersection. At or near the intersection 4 or 5 scan lines will have only a single digitization in the neighborhood of $x_{\text {constan }}$; then $\Delta x$ increases monotonically on the other side of the grid cross mark. It is useful to map out the entire grid in appropriate digitizer coordinates, the intersection point for $x \approx x_{\text {constant }}$, and $y \approx$ the second or third coordinate in the single digitizations near the center. In the $(2,0)$ scan the $y$ coordinate for a fiducial lying vertically above will be larger than for a fiducial directly beneath (see Figure 14). The diagram shows the approximate relationship for a single 5 - $\mathrm{mm}$ grid square ( 1 y unit $=$ $10 \mu, 1 x$ unit $=4.2 \mu$ ). For the $(6,4)$ scan the situation is similar (Figure 15).

The entire grid may be reconstructed as it appears in the dump by stacking 5 -mm squares together. The scan lines are only approximately at $45^{\circ}$; hence diagonal corners of the squares do not have precisely the same $y$ coordinate pictured.

To accurately determine an isosceles right triangle, say $15 \mathrm{~mm}$ per leg, use the following procedure: Take 16 pairs of points on either side of the approximate fiducial center (this gives a consensus of all lenses). Average the $x^{\prime}$ 's; then the.

$$
x \text { center }=\frac{1}{32} \sum_{\mathrm{i}=1}^{32} x_{\mathrm{i} .} .
$$

Although the individual lens coordinates may be in disagreement up to $50 \mu$, the $\Delta x$ among the lenses is rarely in disagreement by $>4 \mu$. Hence, being similar triangles,

$$
y_{\text {center }}=y_{1}+\frac{\Delta x_{1}}{\Delta x_{1}+\Delta x_{2}}\left(y_{2}-y_{1}\right),
$$

where $y_{1}$ and $y_{2}$ are two scan lines approximately equally spaced from the apparent center (as indicated in Figure 16 by the single digitizations in the neighborhood of the center). For several triangles, the actual cycloidal paths are closely approximated by $45^{\circ}$ lines. The results of this procedure may be double-checked for fiducials on the same horizontal level: by using the linear property of a line and the fact that for small slopes, small changes in $x$ over the length of a horizontal line are nearly linear on the cycloid, any one fiducial may be found to within a unit $(4 \mu)$ by interpolation or extrapolation of two other fiducials. For example, the fiducial midway between two others should be represented by the average of their centers. A $15-\mathrm{mm}$ right triangle is simply three corners from a 15-mm square. 


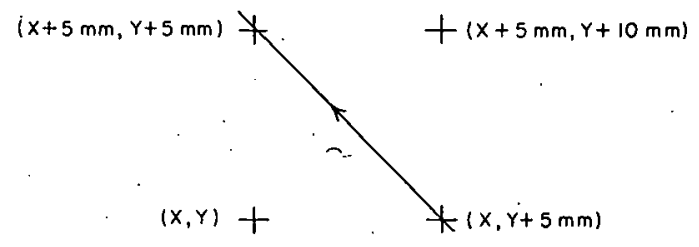

Figure 14.

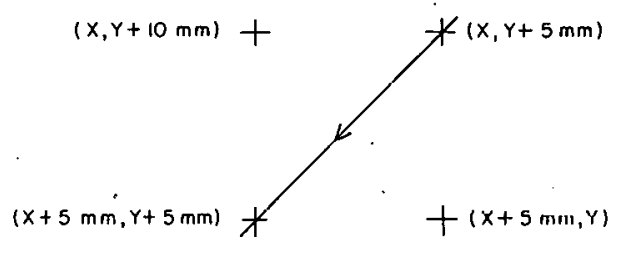

Figure 15.

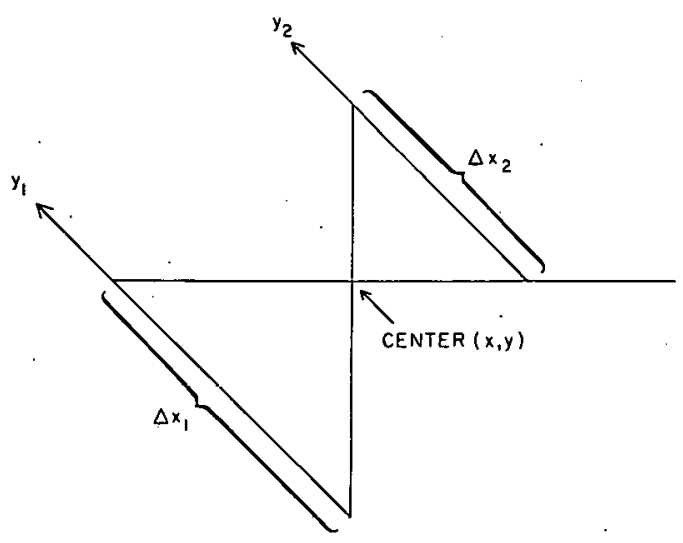

Figure 16.

\section{Determination of Magnification Factors}

Let $F_{1}, F_{2}$ be the magnification factors for the $(2,0)$ and $(6,0)$ scans respectively, i.e., $F_{1} x$ or $F_{2} x$ standardizes an $x$ unit to $4.233 \mu$ measured on a $45^{\circ}$ line to the horizontal ( $y$-axis). Apply the follow: ing formulas, guided by the appropriate diagrams. $\beta$ is the angle the horizontal side of the square makes with the horizontal; $x_{3}$ is the $x$ coordinate struck by the scan line (or interpolated scan line) going diagonally across the $15-\mathrm{mm}$ square as it hits the opposite horizontal side of the square. The $30-\mu$ quantity appearing in the formula for $\gamma$ represents scan line spacing, $l$ is the length of a side of the square, ideally $15 \mathrm{~mm}$, but possibly $n \times 5 \mathrm{~mm}$ for some integer $n$.

$$
\sin \beta=\frac{x_{2}-x_{1}}{l} \sin 50^{\circ},
$$

where $50^{\circ} \approx 45^{\circ}+2 \frac{12^{\circ}}{} \times$ number of squares below center of window where the bottom of large square occurs,

$$
\begin{aligned}
\gamma & =\frac{y_{2}-y_{4}+(\beta / 20) \times 30 \mu}{y_{2}-y_{1}} \cdot l \\
t & =\sqrt{l^{2}+(l-\gamma)^{2}} \\
\delta & =\arcsin (l / t) \\
D & =i \cos \left(45^{\circ}+\beta-\delta\right), \\
F_{1} & =D /\left(x_{3}{ }^{*}-x_{2}^{*}\right),
\end{aligned}
$$

where $x_{3}{ }^{*}, x_{2}{ }^{*}$ are the $x$ 's on the scan line closest to $y_{2}$ and on both horizontal sides of the square. (See Figure 17.)

Similarly,

$$
\sin \beta=\frac{x_{2}-x_{1}}{l} \sin 40^{\circ},
$$

where $40^{\circ} \approx 45^{\circ}-2 \frac{1}{2}{ }^{\circ} \times$ number of 5 -mm squares above center of window where the highest horizontal line for the large square occurs, 


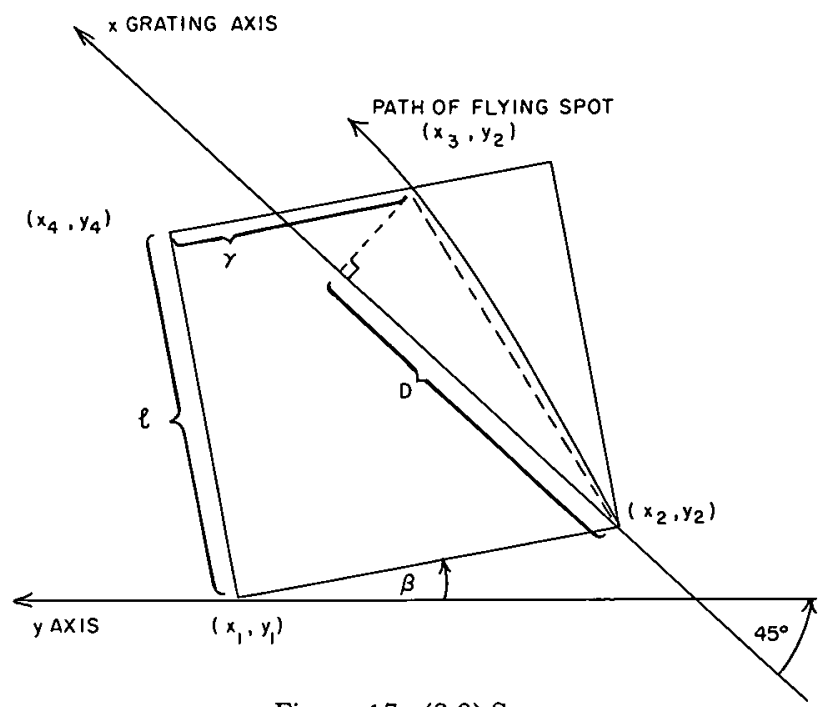

Figure 17. (2,0) Scan.

$$
\begin{aligned}
\gamma & =\frac{y_{4}-y_{1}+(\beta / 20) \times 30 \mu}{y_{2}-y_{1}} l \\
t & =\sqrt{l^{2}+(l-\gamma)^{2}} \\
\delta & =\arcsin (l / t) \\
D & =t \cos \left(45^{\circ}-\beta-\delta\right) \\
F_{2} & =D /\left(x_{3}{ }^{*}-x_{1}^{*}\right)
\end{aligned}
$$

where $x_{3}{ }^{*}, x_{1}{ }^{*}$ are the $x$ 's on the scan line closest to $y_{1}$ and on both horizontal sides of the square. (See Figure 18.)

From the formula of transformation to rectangular coordinates in the $(2,0)$ scan (see ref. 3 ):

$$
\begin{aligned}
& x_{T}=x_{1} \sin \alpha+\cos \alpha\left(R \cos \theta_{2}-M\right), \\
& y_{T}=y_{1}-x_{l} \cos \alpha+\sin \alpha\left(R \cos \theta_{2}-M\right)+\theta p v / 2 \pi
\end{aligned}
$$

The distance $D$ betwcen two points $\left(x_{11}, y_{11}\right)$ and $\left(x_{12}, y_{12}\right)$ is $\sqrt{(D x)^{2}+(D y)^{2}}$, where.

$$
\begin{aligned}
D x & =x_{T 1}-x_{T 2} \\
& =\left(x_{f 1}-x_{f 2}\right) \sin \alpha+R \cos \alpha\left[\sqrt{1-\left(\frac{L-x_{f 1}}{R}\right)^{3}}-\sqrt{1-\left(\frac{L-x_{f 2}}{R}\right)^{2}}\right], \\
D y & =y_{r 1}-y_{T 2} \\
& =y_{f 1}-y_{/ 2}-\left(x_{f 1}-x_{f 2}\right) \cos \alpha+\dot{R} \sin \alpha\left[\sqrt{1-\left(\frac{L-x_{11}}{R}\right)^{2}}-\sqrt{1-\left(\frac{L-x_{f 2}}{R}\right)^{2}}\right]+\frac{\Delta \theta p v}{2 \pi},
\end{aligned}
$$

where

$$
\Delta \theta=\arcsin \frac{x_{f 2}-x_{f 1}}{R} .
$$

The dependence of $D$ on small changes of $R, L$, or $\alpha$ is therefore represented approximately by 


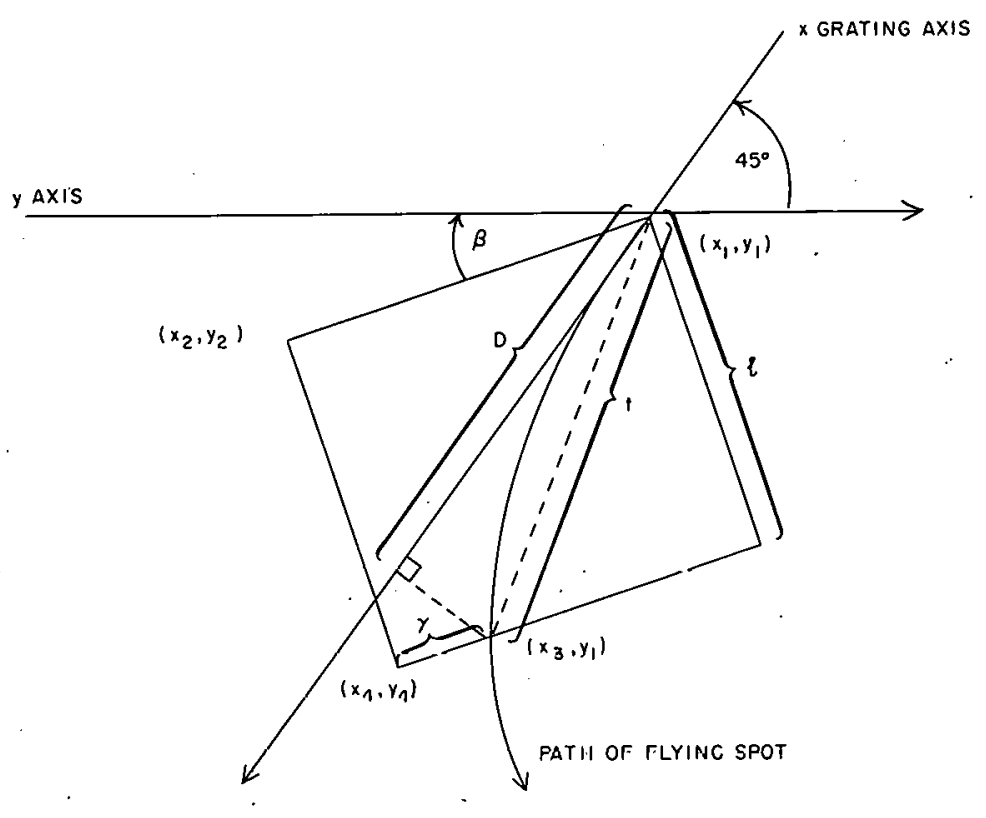

Figure 18. (6,4) Scann.

$$
\begin{aligned}
& \Delta D x=\frac{\partial D x}{\partial R} \Delta R+\frac{\partial D x}{\partial L} \Delta L+\frac{\partial D x}{\partial \alpha} \Delta \alpha, \\
& \Delta D y=\frac{\partial D y}{\partial R} \Delta R+\frac{\partial D y}{\partial L} \Delta L+\frac{\partial D y}{\partial \alpha} \Delta \alpha,
\end{aligned}
$$

where

$$
\begin{aligned}
& \frac{\partial D x}{\partial R} \Delta R \approx \cos \alpha \frac{\left(x_{f 1}-x_{f 2}\right)\left(x_{f 1}+x_{f 2}-2 L\right)}{R^{2}} \Delta R \\
& \frac{\partial D x}{\partial L} \Delta L \approx \cos \alpha(\Delta L)\left[\frac{x_{f 1}-x_{f 2}}{R}+\frac{x_{f 1}{ }^{3}-x_{f 2}{ }^{3}}{2 R^{3}}+\frac{3 L}{2 R^{3}}\left(x_{f 2}{ }^{2}-x_{f 1}{ }^{2}\right)+\frac{3}{2} \frac{L^{2}}{R^{3}}\left(x_{f 1}-x_{f 2}\right)\right], \\
& \frac{\partial D x}{\partial \alpha} \Delta \alpha \approx \Delta \alpha\left[\left(x_{f 1}-x_{/ 2}\right) \cos \alpha-\frac{\left(x_{f 1}-x_{f 2}\right)\left(x_{f 1}+x_{f 2}-2 L\right)}{R} \sin \alpha\right] \\
& \frac{\partial D y}{\partial R} \Delta R \approx \sin \alpha \frac{\left(x_{f 1}-x_{f 2}\right)\left(x_{f 1}+x_{f 2}-2 L\right)}{R^{2}} \Delta R, \\
& \frac{\partial D y}{\partial L} \Delta L \approx \sin \alpha(\Delta L)\left[\frac{x_{f 1}-x_{f 2}}{R}+\frac{x_{f 1}{ }^{3}-x_{f 2}{ }^{3}}{2 R^{3}}+\frac{3 L}{2 R^{3}}\left(x_{f 2}{ }^{2}-x_{/ 1}{ }^{2}\right)+\frac{3}{2} \frac{L^{2}}{R^{3}}\left(x_{f 1}-x_{f 2}\right)\right] \\
& \frac{\partial D y}{\partial \alpha} \Delta \alpha \approx \Delta \alpha\left[\left(x_{f 1}-x_{f 2}\right) \cos \alpha+\frac{\left(x_{f 1}-x_{f 2}\right)\left(x_{f 1}+x_{f 2}-2 L\right)}{R} \cos \alpha\right] .
\end{aligned}
$$

With respect to the formula for $F_{1}$, we have

$$
F_{1}=\frac{D}{D+\Delta D}
$$

where

$$
\Delta D= \pm\left(\sqrt{(D x+\Delta D x)^{2}+(D y+\Delta D y)^{2}}-D\right),
$$

and the two points are the corner of the square $\left(x_{1}, y_{1}\right)$ and the intersection of the $x_{\text {, axis through that }}$ point with the opposite horizontal side of the square. Similar formulas hold for the $(6,4)$ scan. 
The object of the calibration is therefore to get $F_{1}=F_{2}=1$ for any set of triangles chosen. This involves making a crude guess for $R, L$, and $\alpha$, and then inspecting the output from the program VARY which prints out distances between points. Applying the above formulas for $\Delta D$ gives a crude approximation of how $\Delta R, \Delta L$, and $\Delta \alpha$ may be added to or subtracted from $R, L$, and $\alpha$ to arrive at the correct distances between points. However, the solution for one triangle is not usually unique (perhaps any two of the three increments $\Delta R, \Delta L$, and $\Delta \alpha$ will account for $\Delta D$ ). The problem can be viewed in terms of the scanner coordinates directly from the formulas leading to $F_{1}$. In these formulas $\alpha=45^{\circ}$ and $\beta$ is assumed to be correctly determined. Actually both $R$ and $L$ affect $\beta . D+\Delta D$ could then be represented as $t \cos (\alpha+\beta+\Delta \beta-\delta)$, so that $F_{1}=(D+\Delta D) /(D+\Delta D)=1$. The change in the argument of the cosine can be due to either $\alpha$ or $\Delta \beta$, and the same problem as with rectangular coordinates persists. A good initial guess is to distribute the difference $d=(\alpha+\beta+\Delta \beta-\delta)-$ $\left(45^{\circ}+\beta-\delta\right)$ equally, that is, put $\alpha=45^{\circ}+d / 2$ and $\Delta \beta=d / 2$, and thereby get a first estimate for $\alpha$. A finer determination can then be made by changing the ranges of $R, L$, and $\alpha$ in the VARY program and testing the consistency of results over several triangles.

\section{ACKNOWLEDGMENTS}

The authors would like to thank the many people in the Physics Department and its mechanical design section who cooperated in the development of the spark chamber reader system.

\section{REFERENCES}

1. G. Schwender, Report on the Logical Description of the Spark Chamber Reader, BNL 877 (T-35.3), June 1964.

2. G. Schwender, D. Engelberg, R. Schuman, and R. Souires, Report on the Logical Description of the Film Preselector System for the Spark Chamber Reader, BNL 876 (T-352), June 1964.

3. S. Heller, J. Malament, L. Padwa, and M. Strongson, Sofliware for a Spark Chamber Reader System, BNL $977(\mathrm{~T}-412)$, in press. 